Anat Rec (Hoboken). 2012 September ; 295(9): 1455-1461. doi:10.1002/ar.22523.

\title{
Mitochondrial Division in Rat Cardiomyocytes: An Electron Microscope Study*
}

\author{
Hisashi Fujioka1 ${ }^{1}$, Bernard Tandler ${ }^{2}$, and Charles L. Hoppel ${ }^{3}$ \\ ${ }^{1}$ Electron Microscopy Facility and Center for Mitochondrial Disease, School of Medicine, Case \\ Western Reserve University, Cleveland, Ohio \\ 2Department of Biological Sciences, School of Dental Medicine, and Center for Mitochondrial \\ Disease, School of Medicine, Case Western Reserve University, Cleveland, Ohio \\ ${ }^{3}$ Center for Mitochondrial Disease, and Departments of Pharmacology and of Medicine, School of \\ Medicine, Case Western Reserve University, Cleveland, Ohio
}

\begin{abstract}
In cardiomyocytes of rats, two distinct mitochondrial division processes are in operation. The predominant process involves extension of a single crista until it spans the full width of a mitochondrion. Ingrowth of the outer membrane ultimately results in scission. The second division process involves "pinching", in which narrowing of the organelle at specific surface locations leads to its attenuation. When limiting membranes from opposite sides meet, mitochondrial fission ensues. When pinching is the operative mode, elements of sarcoplasmic reticulum always are associated with the membrane constrictions. The nuclear control mechanisms that determine which modality of mitochondrial division will prevail are unknown.
\end{abstract}

\section{Keywords}

mitochondrial division; mitochondrial pinching; cardiac; heart

\section{Introduction}

That mitochondria supply the ATP necessary for muscle cell contraction is a universally accepted truism. Although myocytes do not divide, their mitochondria are relatively shortlived, with a half-life of 5.6 (Gross, 1971) to 6.2 days (Aschenbrenner et al., 1970) for those in cardiac muscle and about one week for those in skeletal muscle (Terjung, 1979). To maintain cell function, the number of mitochondria must be kept at a near constant level. The replenishment of muscle mitochondria appears to be by organelle division. This communication describes two morphological pathways whereby this mitochondrial fission is brought about in cardiomyocytes.

\section{Materials and Methods}

The animals used in this study-- all obtained from the National Institutes of Aging colony (Harlan Sprague-Dawley, Inc., Indianapolis, IN)-- were adult (six months-old) and elderly (31 months-old) Fischer 344 X Brown Norway hybrid rats and adult (six months-old) and

\footnotetext{
* This work was presented in part at the annual meeting of the United Mitochondrial Disease Foundation, Scottsdale AZ, 2010.

Corresponding author: Hisashi Fujioka, PhD, Institute of Pathology Rm. 114, School of Medicine, Case Western Reserve University, 2085 Adelbert Road, Cleveland, OH 44106. Phone 216-368-2490. hisashi.fujioka@ case.edu.
} 
elderly (24 months-old) Fischer 344 rats. The animal studies were approved by the Institutional Animal Care and Use Committees of the School of Medicine, Case Western Reserve University, and of the Louis Stokes Cleveland Department of Veterans Affairs Medical Center. The rats were allowed food and water ad libitum, and were maintained on a 12 hour light:dark cycle. They were killed by decapitation and specimens of left ventricle removed. Samples of the left ventricle were immediately fixed by immersion in the triple aldehyde-DMSO mixture of Kalt and Tandler (1971). After rinsing in distilled water, the tissue blocks were postfixed in ferrocyanide-reduced osmium tetroxide (Karnovsky, 1971). After again rinsing in distilled water, they were soaked overnight in acidified ranyl acetate (Tandler, 1990). Another rinse in distilled water was followed by dehydration in ascending concentrations of ethanol, passage through propylene oxide, and embedment in Poly/Bed resin. Thin sections were sequentially stained with acidified uranyl acetate (Tandler, 1990) followed by Sato's triple lead stain as modified byHanaichi et al. (1986) and examined in a JEOL 1200EX electron microscope.

Pellets of isolated subsarcolemmal (SSM) and of interfibrillar mitochondria (IFM) were prepared as described previously byPalmer et al. (1977) and by Chen et al. (2007). These initially were fixed in eighth-strength Karnovsky's fixative (1965); from this point on, the pellets were treated in the same fashion as the solid tissues, as described above.

\section{Results}

As in virtually all studied mammals, the abundant mitochondria in the rat hearts are aligned in parallel rows between myofibrils, as well as in rows or clusters just beneath the sarcolemma. The vast majority of these organelles have the form of oblate spheroids with numerous transversely-oriented cristae. The abundance of such mitochondria in the cardiomyocytes guarantees that they are closely appressed, but the double membranes of the propinquitous organelles remain readily discernible. We encountered no examples of mitochondria in autophagic vacuoles. There were no obvious qualitative or quantitative differences in the cardiomyocytes of the adult and aged rats, so the mitochondria that we describe herein are not identified as to source. Some rare cardiac mitochondria reveal the formation of a membranous partition that separates a given organelle into two distinct compartments (Fig. 1). Such organelles are found in both subsarcolemmal (SSM) (Fig. 2) and interfibrillar (IFM) (Fig. 3) positions. The direct continuity of the partition with the boundary membrane is shown at high resolution in the inset to Fig.2. Ingrowth of the outer membranes ultimately could lead to mitochondrial fission. Cristae sometimes are slightly reoriented on opposite sides of the partition, but do not assume the orthogonal relationship seen in dividing mitochondria in hearts of cuprizone-treated mice (Tandler and Hoppel, 1972).

A less common (our impression) type of mitochondrial division in the cardiomyocytes involves the "pinching" phenomenon. In this case, the outer membranes on opposite sides of a given interfibrillar mitochondrion make deep incursions into the organelle (Figs. 4, 5). If the intruding antipodal membranes eventually make contact, fission would result. There is a consistent relationship between elements of sarcoplasmic reticulum (SR) and the sites of the invasive mitochondrial membranes (Fig. 5). No constrictions of interfibrillar mitochondria were observed that lacked accompanying SR.

Isolated mitochondria from cardiac muscle were typical in appearance. Scattered among these spherical organelles there was a very small number of partitioned mitochondria (Fig.6 and inset). Such mitochondria are shown at higher magnification in Figs. 7 and 8. The direct continuity of the partitions with the boundary membranes is quite clear. Fig. 8 illustrates an isolated mitochondrion in which the cleavage process is near completion. 
We attempted to ascertain the frequency of partitioned mitochondria in the in situ cardiomyocytes. Using the field shown in Fig. 1 as a starting point, we carefully scanned the five fields to either side of this area for a total of ten fields and were unable to detect another obvious example of a dividing mitochondrion. This exercise was repeated several times with the same result. Because the pellets of isolated mitochondria contain these organelles in a concentrated form, we carried out the same procedure on sequences of ten fields, five on either side of one in which a dividing mitochondrion was observed, again with no further examples of partitioned mitochondria found. Counting of organelles was simplified in pellets of isolated cardiac mitochondria. In such preparations, we found exactly one dividing mitochondrion in $>2,000$ mitochondria. This undoubtedly is an underestimate, since partitioned mitochondria can be identified in thin sections only if the partitions are sectioned near normal. A tilt of only a few degrees from the perpendicular assures that bona fide partitions would be mistaken for mundane cristae. Because some isolated mitochondria at survey magnifications appear to be partitioned, we always checked such possibly dividing organelles at higher magnification to ascertain whether or not these indeed were in the process of division. In most cases such putative fissioning mitochondria did not exhibit true partitions (cf, Fig. 6).

\section{Discussion}

The cardiac muscle cells of untreated adult rats that we examined displayed the two types of mitochondrial morphology that have been shown to be the exemplars of fission. The first of these employs partition formation. Mitochondria of such appearance were originally described in hepatocytes more than half a century ago (Fawcett, 1955), but it could not be decided whether this morphology was consistent with division or fusion or both. The direction of this process was not made clear until the publication of a study of recovering giant mitochondria (Tandler et al., 1969). In this experiment, hepatic mitochondria that had achieved giant size (larger than the hepatocyte nuclei) due to dietary riboflavin deficiency (Tandler et al., 1968) were administered the missing vitamin. The megamitochondria quickly were restored to normal size (within 48 hours) by forming buds with a partition at their base, followed by ingrowth of the outer membrane into the said partition.

That partitions are directly involved in mitochondrial division in a normal, non-experimental setting was demonstrated by Larsen (1970), who investigated the fat body of the skipper butterfly, Calpodes ethlius. Towards the end of the penultimate stage of metamorphosis in this insect, the mitochondria of the fat body (analogous to the liver of mammals) are nearly wiped out by autophagy. Early in the adult stage, the mitochondrial population is restored by mitochondrial division. During the short time when this population is burgeoning, a profusion of mitochondria in all stages of partition formation is observable.

As far as cardiac mitochondria are concerned, Tandler and Hoppel (1972) described a similar mode of division in hearts of mice treated with the copper chelator, cuprizone, which results in a significant increase in the number of mitochondria. This observation is supported by the biochemical study of Medeiros and Jennings (2002). In the mouse heart, the dividing mitochondria exhibit a peculiar arrangement of cristae which, in many cases are seen in profile on one side of the partition, and en face on the other side. The significance of this orthogonal arrangement is unclear.

The aforementioned treatment of mice with cuprizone has an additional effect. Hepatic mitochondria become very large (Suzuki, 1969), some exceeding the nuclei in dimensions. These megamitochondria can be restored to a normal size (with a concomitant increase in mitochondrial number) in a surprisingly short time simply by removing the cuprizone from the diet (Tandler and Hoppel, 1973). Early in recovery, the enlarged mitochondria become 
pleomorphic, then begin to divide by medial attenuation (a form of what is now called pinching).

Interestingly, during reduction of the ariboflavinosis-induced giant hepatic mitochondria only partition formation was involved, whereas reduction of the cuprizone-induced hepatic megamitochondria involved only pinching. In other words, the two modalities of organelledivision never overlapped. In contrast, both types of mitochondrial division were seen in a single cardiomyocyte in our aged rats, although partition formation appeared to be the sole division modality in SSM, with both methodologies occurring in the IFM, although pinching predominated in this site. Whatever nuclear control mechanisms exist (Scarpulla, 1997) to regulate the number of mitochondria in heart cells, the two fission methodologies that we are aware of in the present study were operating simultaneously.

The partitioned mitochondria observed by us in the rat cardiac muscle differed in one respect from those in heart cells of cuprizone-fed mice, namely, the cristae on opposite sides of the septum are not perpendicular to each other, although a different density in the two subcompartments might manifest itself in isolated partitioned mitochondria. The partitions in dividing mitochondria in cardiac muscle are resistant to the rigors of mitochondrial fractionation, as illustrated in Figs. 6 - 8. The slight swelling of such isolated organelles, as well as the loss of matrix density, make the relationship between the partitions and the boundary membrane very clear.

To be sure, partitioned mitochondria might unwittingly be interpreted as representing fusion of propinquitous organelles. That this is not the case was shown by the aforementioned studies where partitioned mitochondria were present in abundance when giant mitochondria were undergoing extreme reduction in size or where the number of mitochondria was exploding. If partitions represented fusion, then giant mitochondria would have assumed gargantuan proportions and the number of mitochondria would have severely decreased, the precise opposite of what actually occurred.

Based on our attempts to quantitate mitochondrial partitioning, it can be concluded that either only a very few cardiac mitochondria are dividing by means of partitions at any given moment or that the process is extremely fast [studies in certain tissue culture cell lines indicate that mitochondria go through $\sim 5$ fission:fusion:division events per hour (Twig et al. 2008)], so the chance of catching a cardiomyocyte mitochondrion in a partitioning mode of division in a thin section is extremely rare.

A consistent finding in our rats was an association between SR and the pinching process, a process strongly believed to be the key step in mitochondrial division (Yoon and McNiven, 2001). As its main function, the SR releases and sequesters calcium, which acts as the trigger for muscle contraction (reviewed by Franzini-Armstrong, 1999). But the SR may have other, not yet characterized, functions (Michalak and Opas, 2009).Volpe et al. (1992) have shown that markers of rough endoplasmic reticulum (RER) are present in the SR of muscle fibers. It has been found that in certain cell types, dynamin-like proteins, which are intimately involved with the mitochondrial division process in mammalian cells (Smirnova et al., 2001), are associated with the endoplasmic reticulum (Yoon et al., 1998). It is possible that RER components of the SR synthesize dynamin or a dynamin-like protein and that the SR directs it to specific receptors (Otera and Mihara, 2011) on the outer membrane of the mitochondria, a juxtaposition that may be maintained by mitofusin 2 (de Brito and Scorrano, 2008). Treatment of mice with mitochondrial division inhibitor-1 leads to lengthening of cardiomyocyte mitochondria, presumably by neutralization of Drp1, a dynamin-related protein (Ong et al., 2010). As noted in yeast, Drp1 may form a cincture around the mitochondrion and by constriction sever the organelle (Mears et al., 2007; Detmer and 
Chan, 2007). Secondarily, the SR might conduct calcium to specific points on the outer mitochondrial membrane (Pizzo and Pozzan, 2007), resulting in the recruitment of Drp1, leading to fission of the organelle (Breckenridge et al., 2003). In addition, the SR may act as a conduit for components (proteins, phospholipids, etc.) of the newly developing mitochondrial membranes (Vance, 1990; Kornmann et al, 2009), since surface area to volume ratios dictate that even a typical interfibrillar mitochondrion undergoing pinching (such as that shown in Fig. 3) ultimately will require a significant increase in new membrane to cover the two daughter organelles.

That ER is involved in mitochondria division was recently reported byFriedman et al. (2011). These authors, dealing with yeast cells and cultured Cos cells (monkey kidney fibroblasts), show a propinquity between ER (not identified as to type [rough or smooth endoplasmic reticulum?]) and mitochondria that show a degree of constriction, which are presumed to be in a division mode. Such constrictions are rather broad, and not sharply defined incisures such as those we have shown; our micrographs, which are of in situ cardiac tissue, display SR elements that are deeply inserted into clearly limned notches that have nearly transected the mitochondria. The EM reconstructions and fluorescence microscopy byFriedman et al. (2011) clearly show that belt-like formations of ER are intimately associated with mitochondrial pinching, suggesting division in single cells, and support our contention that this relationship is a crucial one. Although it would be of interest to have information on the frequency of this relationship, our study at the EM level did not readily lend itself to a quantitative approach. Suffice it to say that every mitochondrion that we observed exhibiting any degree of pinching had SR adjacent to membrane incursions. How this ER-mitochondria relationship applies to division by means of partition formation has not been addressed.

Although a great deal is known about the molecular mechanisms of mitochondrial division [reviewed by Chan (2006), by Liesa et al. (2009), and by Lackner and Nunnari (2009)], there is still a host of proteins involved in mitochondrial fission in eukaryotes whose precise role in this process remain to be elucidated. Although it has been established that the nucleus regulates mitochondrial number (reviewed by Rapaport, 2003), nuclear control mechanisms that determine which morphological pathway in mitochondrial division will be activated are unknown and seem to have been little studied by researchers.

\section{Acknowledgments}

This work was supported in part by Program Project Grant 2P01015885 from the NIA. Kiet Luc provided technical assistance. We thank Drs. Jason Mears and Xin Qi for helpful discussions.

\section{LITERATURE CITED}

Aschenbrenner V, Druyan R, Rabinowitz M. Haem $o$ cytochrome $c$ and total protein turnover in mitochondria from rat heart and liver. Biochem J. 1970; 119:157-160. [PubMed: 5488910]

Breckenridge DG, Stojanovic M, Marcellus RC, Shore GC. Caspase cleavage product of BAP31 induces mitochondrial fission through endoplasmic reticulum calcium signals, enhancing cytochrome $c$ release to the cytosol. J Cell Biol. 2003; 160:1115-1127. [PubMed: 12668660]

Chan DC. Mitochondrial fusion and fission in mammals. Annu Rev Cell Dev Biol. 2006; 22:79-99. [PubMed: 16704336]

Chen H, Chan DC. Energizing functions of mammalian mitochondrial fusion and fission. Hum Mol Genet. 2005; 11:R283-R289. [PubMed: 16244327]

Chen Q, Hoppel CL, Lesnefsky EJ. Blockage of electron transport before cardiac ischemia with the reversible inhibitor amobarbital protects rat heart mitochondria. J Pharmacol Exp Ther. 2006; 316:200-207. [PubMed: 16174799] 
de Brito OM, Scorrano L. Mitofusin 2 tethers endoplasmic reticulum to mitochondria. Nature. 2008; 456:605-610. [PubMed: 19052620]

Detmer SA, Chan DC. Functions and dysfunctions of mitochondrial dynamics. Nature Rev Mol Cell Biol. 2007; 8:870-879. [PubMed: 17928812]

Fawcett DW. Observations on the cytology and electron microscopy of hepatic cells. J Nat Cancer Inst. 1955; 15(Suppl.):1475-1502. [PubMed: 13243087]

Franzini-Armstrong C. The sarcoplasmic reticulum and the control of muscle contraction. FASEB J. 1999; 13:S266-S270. [PubMed: 10619141]

Friedman JR, Lackner LL, West M, DiBenedetto JR, Nunnari J, Voeltz GK. ER tubules mark sites of mitochondrial division. 10.1126/science.1207285.

Gross NG. Control of mitochondrial turnover under the influence of thyroid hormone. J Cell Biol. 1971; 48:29-40. [PubMed: 5545111]

Hanaichi T, Sato T, Iwamoto T, Malavasi-Yamashiro J, Hoshino M, Mizuno N. A stable lead by modification of Sato's method. J Electron Microsc. 1986; 35:304-306.

Kalt MR, Tandler B. A study of fixation of early amphibian embryos for electron microscopy. J Ultrastruct Res. 1971; 36:633-645. [PubMed: 4939156]

Karnovsky MJ. A formaldehyde-glutaraldehyde fixative of high osmolality for use in electron microscopy [abstract]. J Cell Biol. 1965; 27:137A-138A.

Karnovsky, MJ. Proc 11th Annu Mtg Am Soc Cell Biol. New Orleans, LA: 1971. Use of ferrocyanidereduced osmium tetroxide in electron microscopy; p. 146

Kornmann B, Currie E, Collins SR, Sculdiner M, Nunnari J, Weissman JS, Walter P. An ERmitochondria tethering complex revealed by a synthetic biology screen. Science. 2009; 325:477481. [PubMed: 19556461]

Lackner LL, Nunnari JM. The molecular mechanism and cellular functions of mitochondrial division. Biochim Biophys Acta. 2009; 1792:1138-1134. [PubMed: 19100831]

Larsen WJ. Genesis of mitochondria in insect fat body. JCell Biol. 1970; 47:373-383. [PubMed: 19866737]

Liesa M, Palacin M, Zorzano A. Mitochondrial dynamics in mammalian health and disease. Physiol Rev. 2009; 89:799-845. [PubMed: 19584314]

Mears JA, Ray P, Hinshaw JE. A corkscrew model for dynamin constriction. Structure. 2007; 15:1190-1202. [PubMed: 17937909]

Medeiros DM, Jennings D. Role of copper in mitochondrial biogenesis via interaction with ATP synthase and cytochrome $c$ synthase. J Bioenerg Biomembr. 2002; 34:389-395. [PubMed: 12539966]

Michalak M, Opas M. Endoplasmic and sarcoplasmic reticulum in the heart. Trends Cell Biol. 19:253259. [PubMed: 19409791]

Ong S-B, Subrayan S, Lim SY, Yellon DM, Davidson SM, Hausenloy DJ. Inhibiting mitochondrial fission protects the heart against ischemia/reperfusion injury. Circulation. 2010; 121:2012-2022. [PubMed: 20421521]

Otera H, Mihara K. Discovery of the membrane receptor for mitochondrial fission GTPase Drp1. Small Gtpases. 2011; 2:167-172. [PubMed: 21776419]

Palmer JW, Tandler B, Hoppel CL. Biochemical properties of subsarcolemmal and interfibrillar mitochondria isolated from rat cardiac muscle. J Biol Chem. 1977; 252:8731-8739. [PubMed: 925018]

Pizzo P, Pozzan T. Mitochondria-endoplasmic reticulum choreography: structure and signaling dynamics. Trends Cell Biol. 2007; 17:511-517. [PubMed: 17851078]

Rapaport D. Finding the right organelle. Targeting signals in mitochondrial outer-membrane proteins. EMBO Rep. 2003; 4:948-952. [PubMed: 14528265]

Smirnova E, Griparic L, Shurland D-L, van der Bliek AM. Dynamin-related protein Drp1 is required for mitochondrial division in mammalian cells. Mol Biol Cell. 2001; 12:2245-2256. [PubMed: 11514614]

Scarpulla RC. Nuclear control of respiratory chain expression in mammalian cells. J Bioenerg Biomemb. 1997; 29:109-119. 
Suzuki K. Giant hepatic mitochondria: production in mice fed with cuprizone. Science. 1969; 163:6162.

Tandler B. Improved uranyl acetate staining for electron microscopy. J Electron Microsc Tech. 1990; 16:81-82. [PubMed: 1698950]

Tandler B, Erlandson RA, Smith AL, Wynder L. Riboflavin and mouse hepatic cell structure and function. II. Division of mitochondria during recovery from simple deficiency. J Cell Biol. 1969; 41:477-493. [PubMed: 5783869]

Tandler B, Erlandson RA, Wynder EL. Riboflavin and mouse hepatic cell structure and function. I. Ultrastructural alterations in simple deficiency. Am J Pathol. 1968; 52:69-95. [PubMed: 4169053]

Tandler B, Hoppel CL. Possible division of cardiac mitochondria. Anat Rec. 1972; 173:309-324. [PubMed: 5039085]

Tandler B, Hoppel CL. Division of giant mitochondria during recovery from cuprizone intoxication. J Cell Biol. 1973; 56:266-272. [PubMed: 4118453]

Terjung RL. The turnover of cytochrome $c$ in different skeletal muscle fiber types of the rat. Biochem J. 1979; 178:569-574. [PubMed: 222256]

Twig G, Hyde B, Shirhai OS. Mitochondrial fusion, fission and autophagy as a quality control axis: the bioenergetic view. Biochim Biophys Acta. 2008; 1777:10921097.

Vance JE. Phospholipid synthesis in a membrane fraction associated with mitochondria. J Biol Chem. 1990; 265:7248-7256. [PubMed: 2332429]

Volpe P, Villa A, Podini P, Martini A, Nori A, Panzeri MC, Meldolesi J. The endoplasmic reticulumsarcoplasmic reticulum connection: distribution of endoplasmic reticulum markers in the sarcoplasmic reticulum of skeletal muscle fibers. Proc Natl Acad Sci USA. 1992; 89:6142-6146. [PubMed: 1631100]

Yoon Y, McNiven MA. Mitochondrial division: new partners in membrane pinching. Curr Biol. 2001; 11:R67-R70. [PubMed: 11231145]

Yoon Y, Pitts KR, Dahan S, McNiven MA. A novel dynamin-like protein associates with cytoplasmic vesicles and tubules of the endoplasmic reticulum and mitochondria in mammalian cells. Mol Biol Cell. 1998; 140:779-793. 

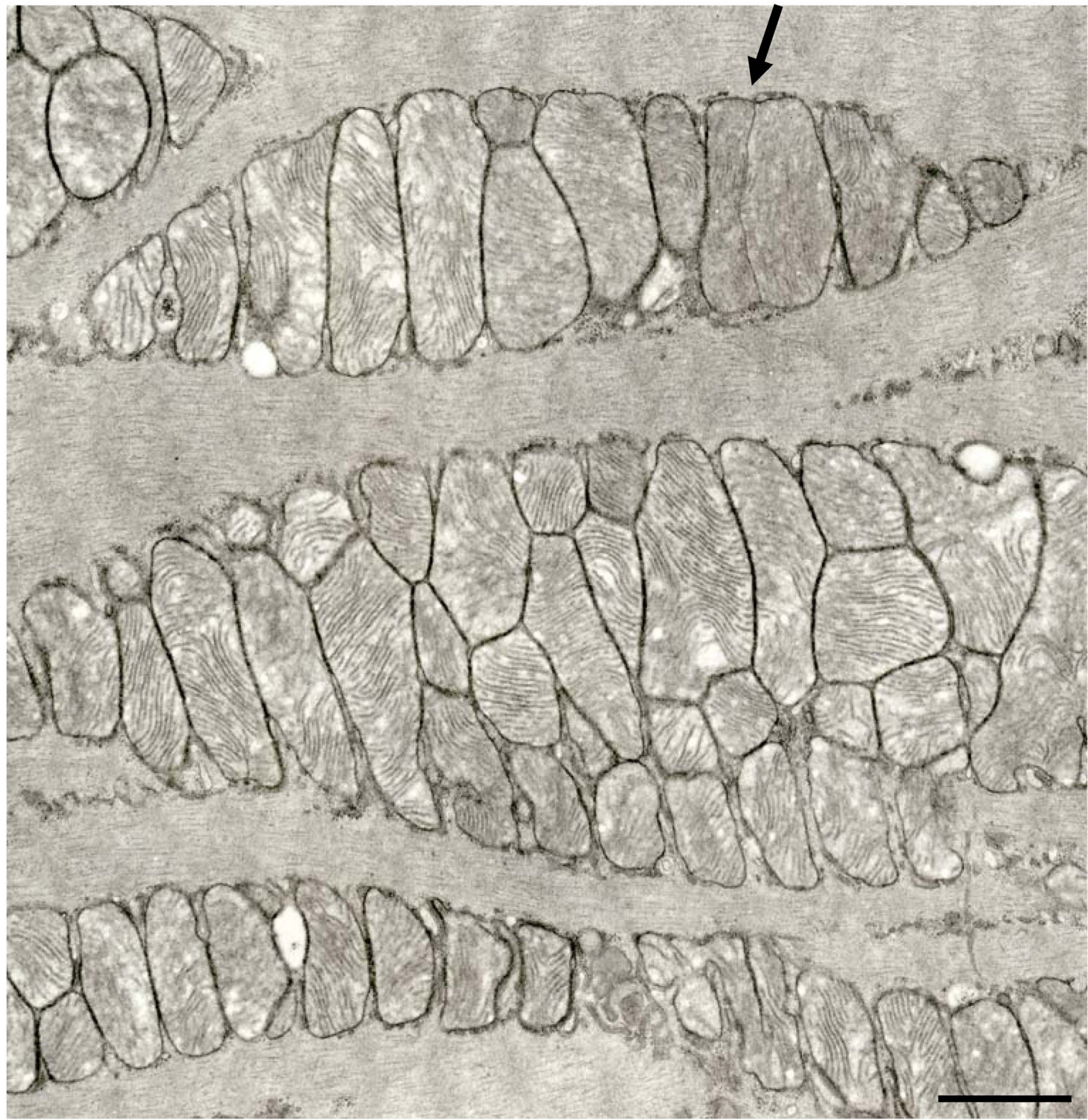

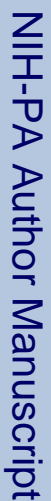

Fig. 1.

Several aggregations of interfibrillar mitochondria. A partitioned mitochondrion is indicated by the arrow. 24 month Fischer 344 rat. Scale bar $=1.0 \mu \mathrm{m}$. 


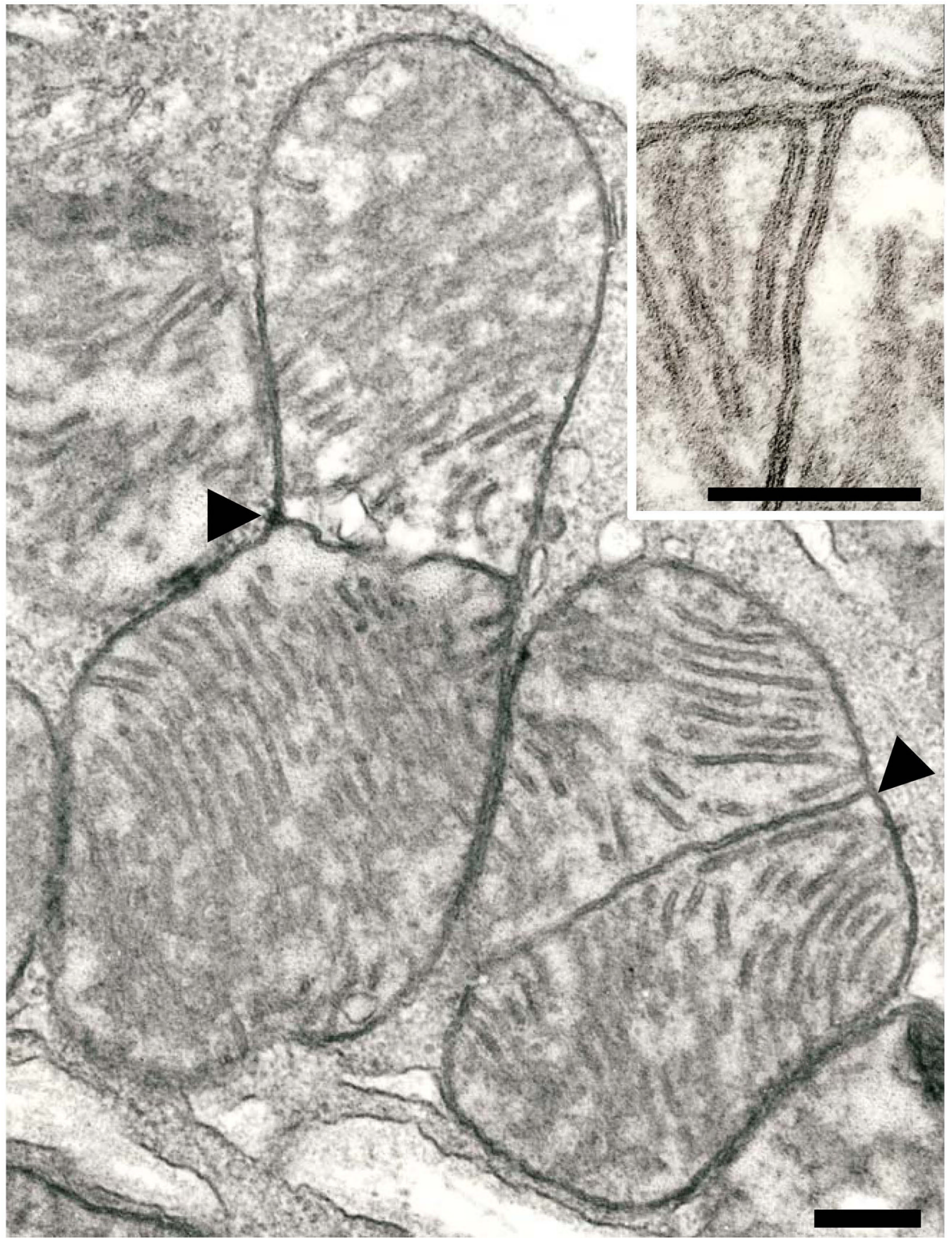

Fig. 2.

A pair of subsarcolemmal mitochondria with medial partitions (arrowheads) in a 24 monthold Fischer 344 rat. Cristae on either side of the partition are seen in profile. Scale bar $=0.2$ $\mu \mathrm{m}$. Inset. The point of connection of a median partition with the boundary membrane. Note that the outer membrane spans this connection. A conventional crista is at the left. The unit membrane structure of all membranes is clearly resolved. 24 month-old Fischer 344 rat. Scale bar $=0.2 \mu \mathrm{m}$. 


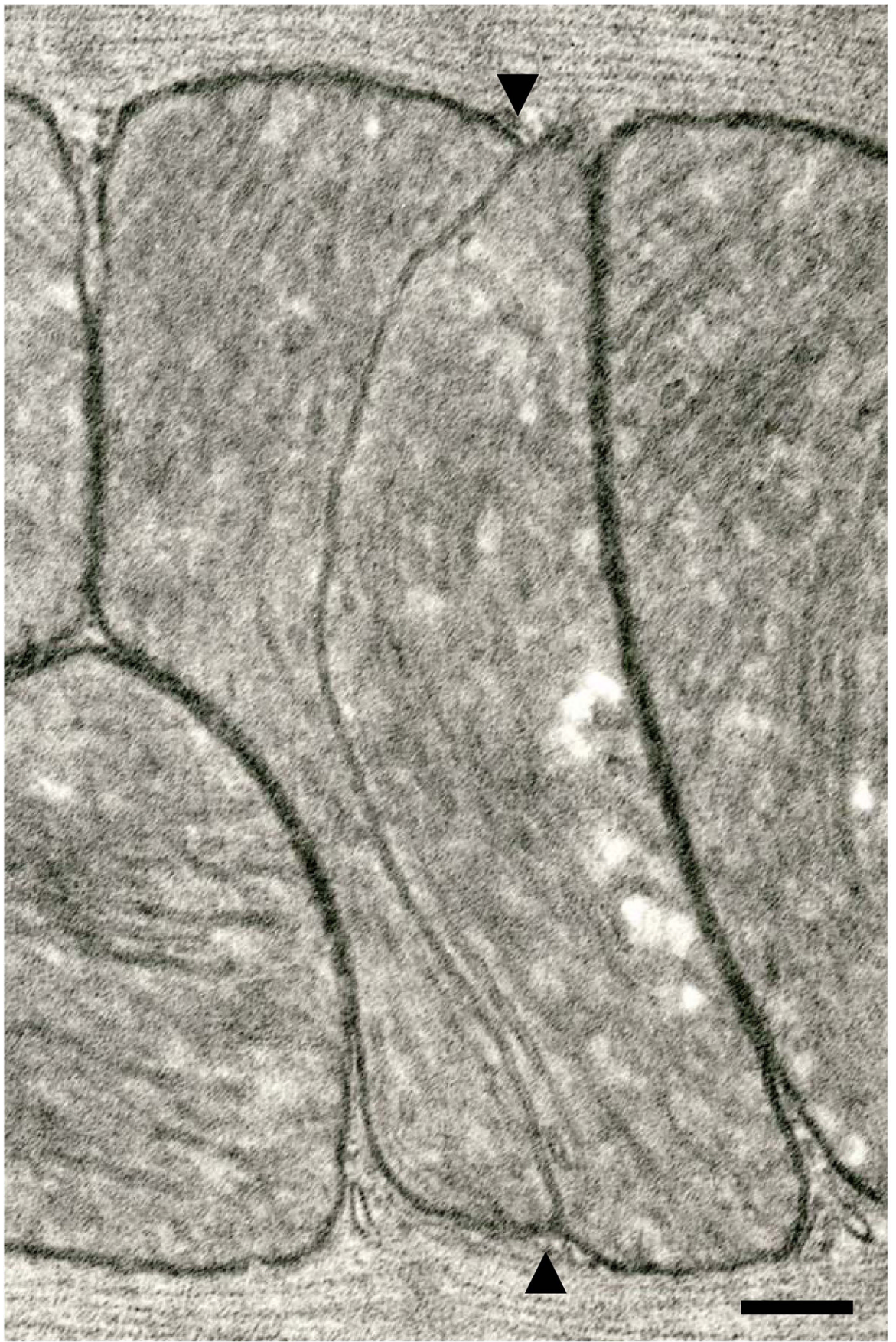

Fig. 3.

An interfibrillar mitochondrion with a longitudinally-oriented median partition (arrowheads). Six month-old Fischer 344 rat. Scale bar $=0.2 \mu \mathrm{m}$ 


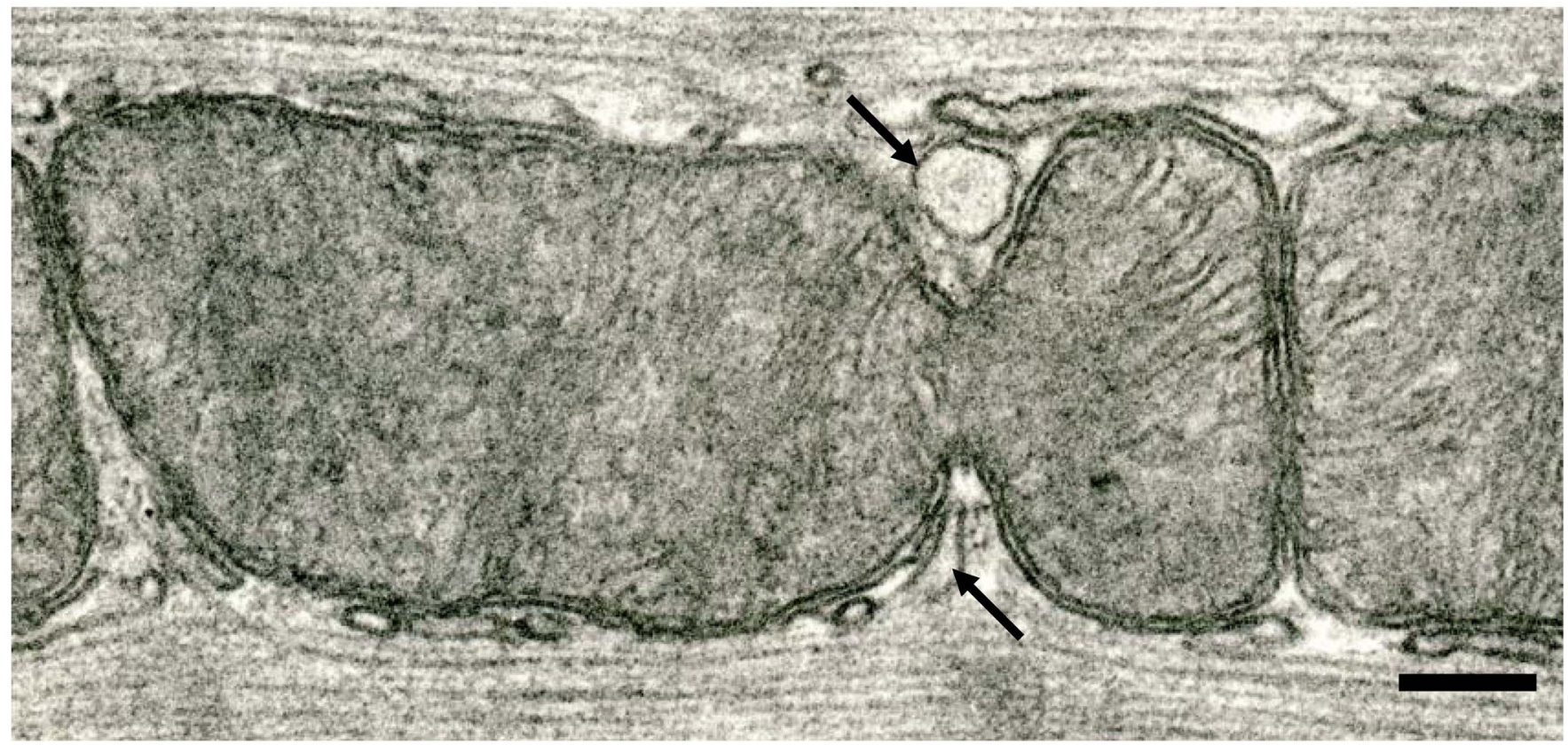

Fig. 4.

An interfibrillar mitochondrion undergoing pinching. Note the elements of SR related to the membrane incursions (arrows). Six month-old Fischer 344 rat. Scale bar $=0.2 \mu \mathrm{m}$ 


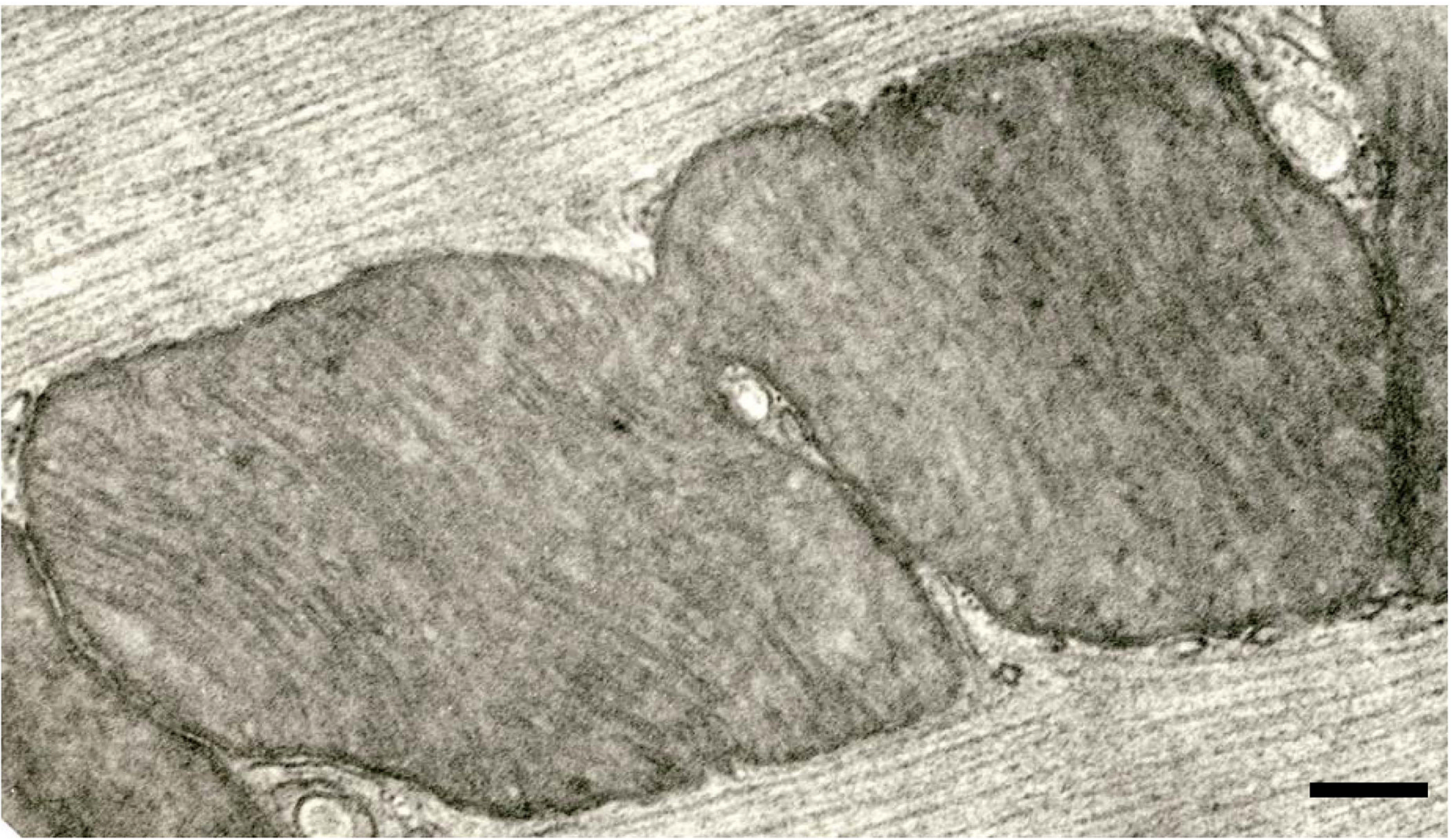

Fig. 5.

An interfibrillar mitochondrion that has almost completed the fission process; the two prospective daughter organelles are still connected by a slender isthmus. An element of SR has penetrated the constriction to its very terminus. Six month-old Fischer 344 rat. Scale bar $=0.2 \mu \mathrm{m}$ 


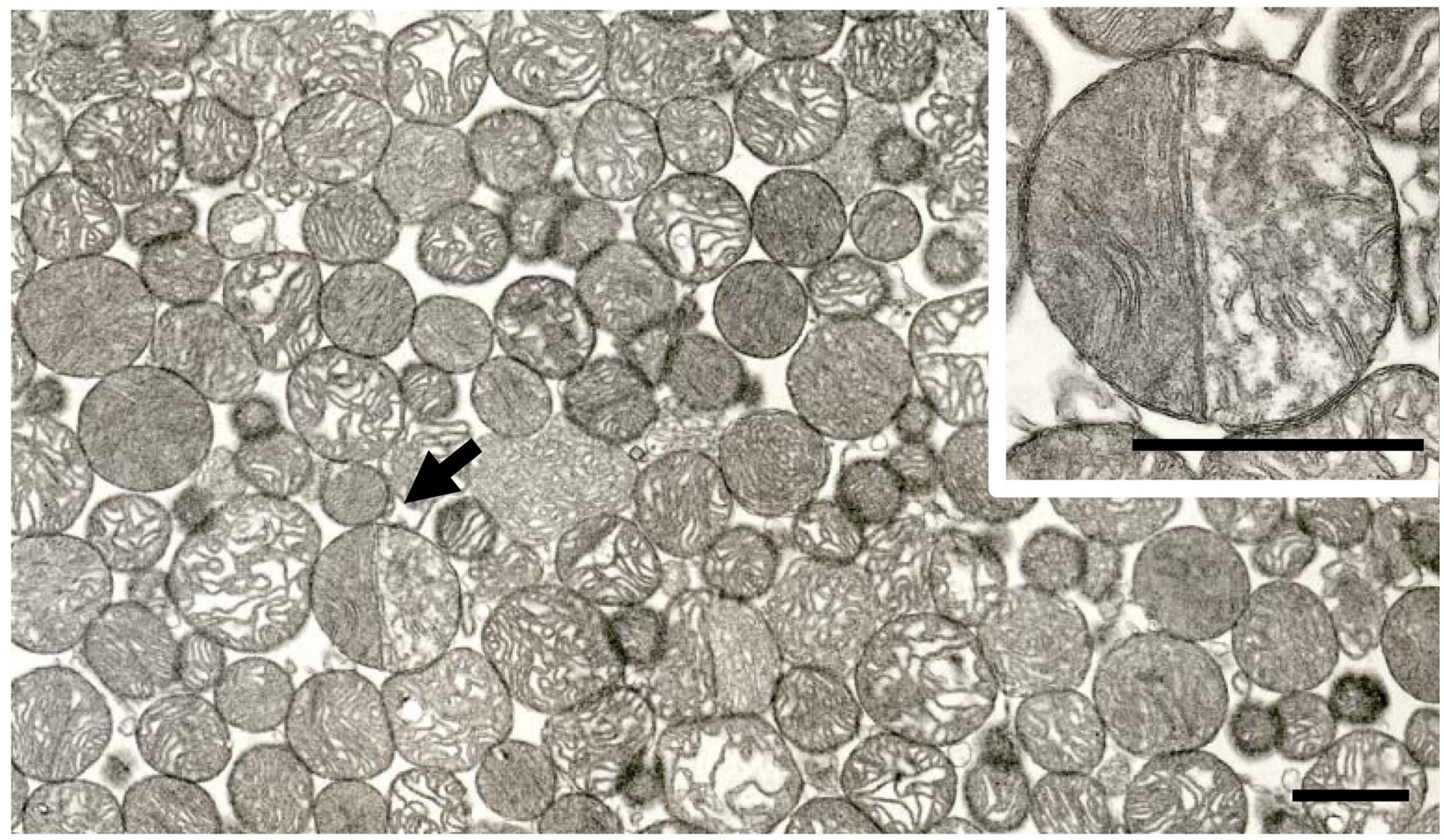

Fig. 6.

Survey micrograph of a pellet of IFM. A partitioned mitochondrion is indicated by the arrow. This organelle is shown at higher magnification in the inset. Although some neighboring mitochondria superficially appear to possess partitions, only the organelle shown in the inset had a partition that met our criteria for such a morphology. Six month-old Fischer 344 rat. Scale bar $=1.0 \mu \mathrm{m}$. Inset. The partitioned mitochondria from Fig 6. The bicompartmentalized structure of this organelle is accentuated by the different density of its two halves, due in part to a different orientation of cristae in its two sides. Scale bar $=1.0$ $\mu \mathrm{m}$. 


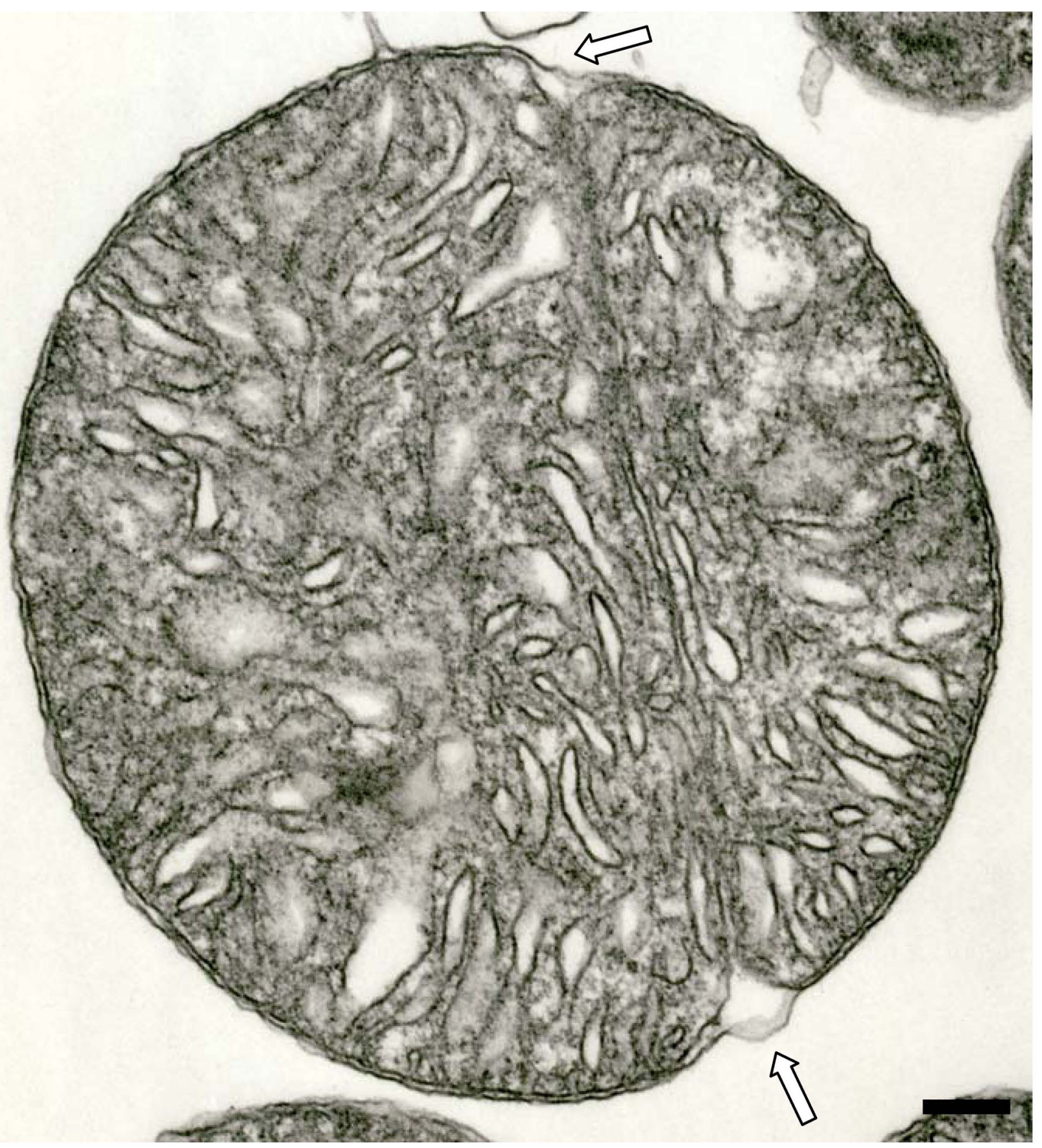

Fig. 7.

An isolated IFM that exhibits a median partition. The outer membrane (open arrows) does not extend into the partition. Six month-old hybrid rat. Scale bar $=0.2 \mu \mathrm{m}$ 


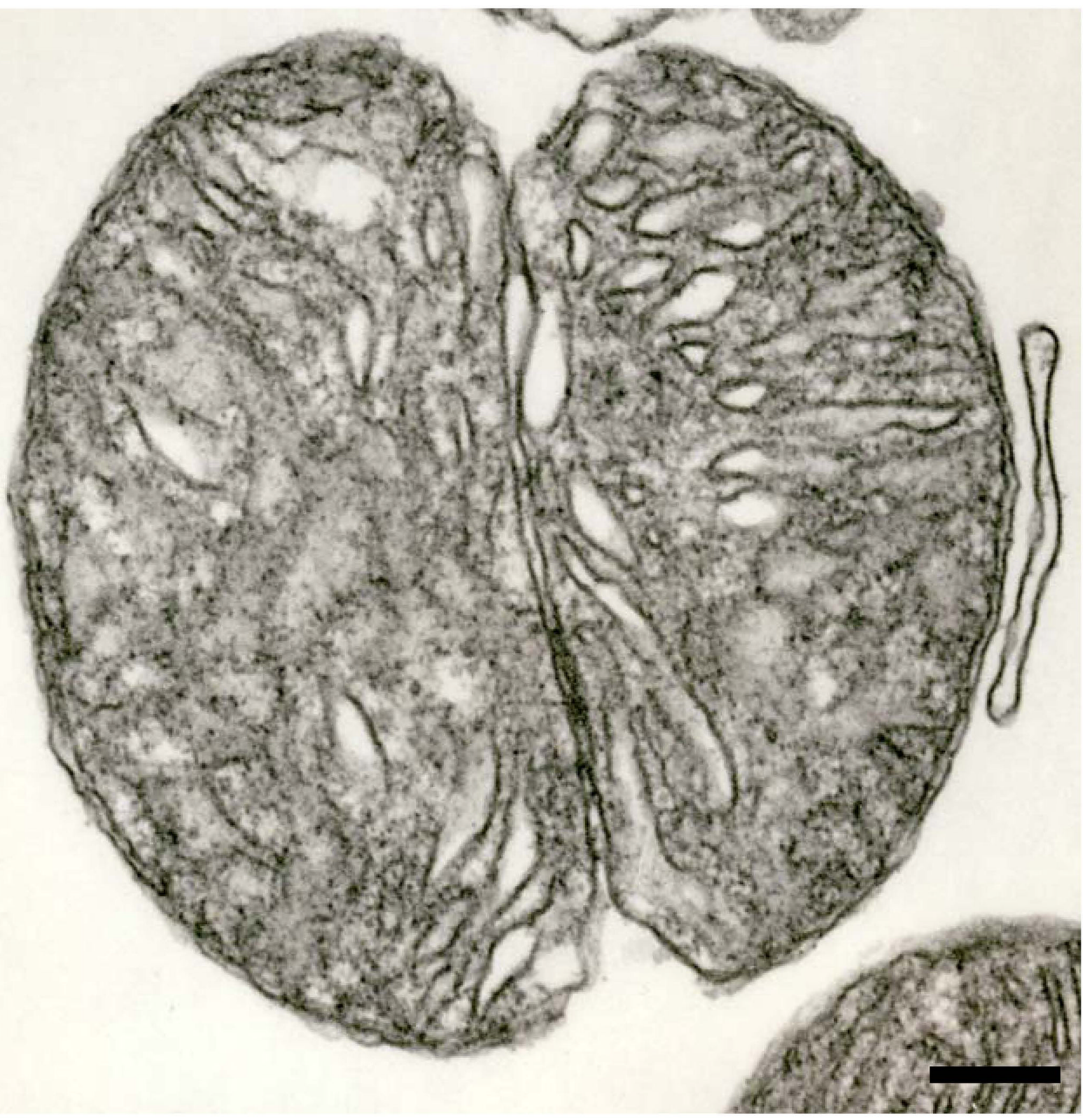

Fig. 8.

An isolated mitochondrion in a more advanced stage of division than that shown in the preceding figure. Continued ingrowth of the outer membrane would lead to the separation of the two daughter organelles. Six month-old hybrid rat. Scale bar $=0.2 \mu \mathrm{m}$ 